\title{
Conhecimento do Enfermeiro da Atenção Primária à Saúde sobre Sexualidade no
}

\section{Climatério}

\author{
Knowledge of Primary Health Care Nurses on Sexuality in Climacteric \\ Conocimientos de Enfermeras de Atención Primaria de Salud sobre la Sexualidad en el Climatérico
}

Recebido: 27/01/2022 | Revisado: 03/02/2022 | Aceito: 09/02/2022 | Publicado: 14/02/2022

\author{
Ângela Roberta Lessa de Andrade \\ ORCID: https://orcid.org/0000-0001-7753-675X \\ Universidade de Pernambuco, Brasil \\ E-mail: angelalessadeandrade@yahoo.com.br \\ Alice Fonseca Pontes \\ ORCID: https://orcid.org/0000-0002-3291-5964 \\ Universidade de Pernambuco, Brasil \\ E-mail: alicepontes136@gmail.com \\ Beatriz Caetano da Silva \\ ORCID: https://orcid.org/0000-0003-4853-1663 \\ Universidade de Pernambuco, Brasil \\ E-mail: beatrizcaetano20001@gmail.com \\ Mirela Ferreira Pessoa Deodoro \\ ORCID: https://orcid.org/0000-0001-9571-3828 \\ Universidade de Pernambuco, Brasil \\ E-mail: mireladeodoro@gmail.com \\ Sara Rodrigues Cordeiro da Silva \\ ORCID: https://orcid.org/0000-0002-4748-8627 \\ Universidade de Pernambuco, Brasil \\ E-mail: sara.rodrigues@upe.br \\ Fátima Maria da Silva Abrão \\ ORCID: https://orcid.org/0000-0003-3254-2851 \\ Universidade de Pernambuco, Brasil \\ E-mail: fatima.abrao@upe.br \\ Ana Maria de Almeida \\ ORCID: https://orcid.org/0000-0002-6398-7194 \\ Universidade de Pernambuco, Brasil \\ E-mail: maria.almeida@saude.gov.br \\ Isabel Cristina Ramos Vieira Santos \\ ORCID: https://orcid.org/0000-0002-5458-4334 \\ Universidade de Pernambuco, Brasil \\ E-mail: tutornad@gmail.com \\ Aurélio Molina da Costa \\ ORCID: https://orcid.org/0000-0002-2641-7686 \\ Universidade de Pernambuco, Brasil \\ E-mail: Aumolina@gmail.com
}

\begin{abstract}
Resumo
Objetivo: analisar o conhecimento do enfermeiro da Atenção Primária à Saúde sobre sexualidade da mulher no climatério e suas interfaces. Método: o presente estudo trata-se de uma revisão integrativa. Foram encontradas 873 publicações nas quais analisou-se os títulos e resumos, como também, os critérios de inclusão e exclusão proposto. Em seguida, 171 publicações foram pré-selecionadas para ser realizada a leitura na íntegra com o intuito de analisar criticamente os resultados obtidos. Após o processo de leitura e análise, 39 publicações foram selecionadas para compor a discussão e apresentação desta revisão integrativa. Resultados e discussão: em análise, foi constatado que a literatura traz a necessidade do profissional de enfermagem trabalhar a temática da sexualidade de forma clara, abordando as necessidades do indivíduo como um todo. Conclusão: desse modo, os achados e reflexões apresentados neste estudo podem contribuir para a melhoria da assistência prestada às mulheres no climatério pelos enfermeiros na Estratégia Saúde da Família.
\end{abstract}

Palavras-chave: Sexualidade; Climatério; Atenção Primária à Saúde; Enfermagem; Estratégia Saúde da Família.

\section{Abstract}

Objective: to analyze the knowledge of Primary Health Care nurses about women's sexuality in climacteric and their interfaces. Method: the present study is an integrative review. 873 publications were found in which the titles and abstracts were analyzed, as well as the proposed inclusion and exclusion criteria. Then, 171 publications were pre- 
selected to be read in full in order to critically analyze the results obtained. After the reading and analysis process, 39 publications were selected to compose the discussion and presentation of this integrative review. Results and discussion: in analysis, it was found that the literature brings the need for the nursing professional to work the theme of sexuality in a clear way, addressing the needs of the individual as a whole. Conclusion: in this way, the findings and reflections presented in this study can contribute to the improvement of care provided to climacteric women by nurses in the Family Health Strategy.

Keywords: Sexuality; Climacteric; Primary Health Care; Nursing; Family Health Strategy.

\section{Resumen}

Objetivo: analizar el conocimiento de enfermeros de la Atención Primaria de Salud sobre la sexualidad de la mujer en climaterio y sus interfaces. Método: el presente estudio es una revisión integradora. Se encontraron 873 publicaciones en las que se analizaron los títulos y resúmenes, así como los criterios de inclusión y exclusión propuestos. Luego, se preseleccionaron 171 publicaciones para ser leídas en su totalidad con el fin de analizar críticamente los resultados obtenidos. Después del proceso de lectura y análisis, 39 publicaciones fueron seleccionadas para componer la discusión y presentación de esta revisión integradora. Resultados y discusión: en el análisis, se constató que la literatura trae la necesidad de que el profesional de enfermería trabaje el tema de la sexualidad de forma clara, abordando las necesidades del individuo como un todo. Conclusión: de esa forma, los hallazgos y reflexiones presentados en este estudio pueden contribuir para la mejora de la atención prestada a la mujer climatérica por los enfermeros en la Estrategia Salud de la Familia.

Palabras clave: Sexualidad; Climatérico; Primeros Auxilios; Enfermería; Estrategia de Salud de la Familia.

\section{Introdução}

Em 1984 foi criado pelo Ministério da Saúde (MS) o Programa de Assistência Integral à Saúde da Mulher (PAISM), que teve como objetivo promover a saúde da mulher no climatério (Brasil, 2008; Brasil, 2011). No Brasil, a pessoa idosa é definida como aquela com idade acima de 60 anos (Santos, 2021; Maciel, et al., 2021). O climatério é marcado por mudanças que caracterizam o término do período reprodutivo (Brasil, 2010). Essa transição lida com as mudanças internas decorrentes das alterações hormonais, como também da perda do potencial reprodutivo e do envelhecimento propriamente dito (Silva et al., 2010).

Ainda com a Silva et al., (2010), os fenômenos fisiológicos são decorrentes de uma da fase natural da mulher, caracterizando o sinal de maturidade. Já na visão de algumas mulheres, essa fase significa perdas marcantes, velhice ou aproximação de doenças. Sendo assim, a sexualidade é identificada como a vivência de pensamentos, desejos, atitudes, valores, comportamentos e relacionamentos (Silva, et al., 2021; Pontes, et al., 2021; Nogueira \& Pachú, 2021). As mulheres são a maioria da população brasileira usuária do Sistema Único de Saúde (SUS).

Em partida, para Zampieri, et al. (2009), parte das dificuldades sexuais está ligada a problemas externos que são projetados e refletidos na vida sexual. Com base em uma sociedade que preza a juventude, para as mulheres que vivem o climatério, entrar na meia idade pode desencadear efeitos emocionais profundos levando muitas vezes a baixa da auto-estima (Zampieri, et al., 2009).

De acordo com Pontes, et al., (2021), o tema da sexualidade humana é um tabu dentro da sociedade, principalmente por parte das mulheres. Ainda com Pontes, et al., (2021), os problemas biopsicossociais e culturais, trazem a complexa conjunção de vida humana, baseada nas etapas reprodutivas e a ligação familiar, autocuidado, saúde física, mental e as relações interpessoais. Socialmente, é comum a presença de problemas sociais, como a saída dos filhos de casa, aparição de doenças, perda de familiares e amigos queridos, e o estresse dos problemas no relacionamento (Melo et al., 2019).

Em relatos de Valença et al., (2010), a sexualidade no climatério abrange não apenas o ato sexual propriamente dito, mas o sentimento, sendo considerado a subjetividade de cada ser humano como diferentes perspectivas e particularidades. Não é de hoje que essa temática de tanta importância vem sendo abordada. Em 1853 Sigmund Freud e Henry Ellis, em 1859, tornaramse pioneiros por começarem a estudar a sexualidade humana (Sena, 2010). Ainda com Sena (2010), de 1894 à 1953, Alfred 
Kinsey teve a realização de um estudo que almejava compreender o comportamento sexual dos americanos com diferentes características de gênero, o que influenciou o então intitulado como "revolução sexual", durante as décadas de 50 e 60 .

Entre 1957 e 1990, os estudiosos Masters e Johnson buscaram compreender a resposta sexual por meio dos aspectos fisiológicos, surgindo assim, a identificação de padrões diferentes o que instigou o tratamento para "desordens sexuais" (Sena, 2010). Com isso, Helen Kaplan utilizou esses estudos como base de aprofundamento metodológico para inserir a psicoterapia como meio de tratamento, dando origem à terapia sexual (Sena, 2010).

Pensando dessa forma, o MS em 2006 incluiu no caderno de Atenção à Pessoa Idosa um capítulo dedicado à sexualidade da mulher idosa, almejando assim a melhoria de abordagem por meio de profissionais da saúde referente à sexualidade no climatério (Brasil, 2006).

No ano de 2010, o MS realizou a publicação de um caderno destinado à Atenção Primária em Saúde Sexual e Reprodutiva, que desde então vem sendo uma das muitas referências na área da sexualidade humana de grande relevância (Brasil, 2010). De acordo com Cruz da Silva, et al. (2013), o enfermeiro ainda tem dificuldades em trabalhar a temática por consequência de uma falha ainda na graduação, que é a falta de abordagem sobre as interfaces da sexualidade como um todo, criando uma lacuna específica na formação profissional.

Em outros estudos, o conhecimento acadêmico de enfermagem sobre sexualidade concluíram que existe a necessidade do fortalecimento da educação continuada e da inclusão de uma disciplina voltada à sexualidade, trabalhada de forma transversal a outras disciplinas, visando melhoria do profissional (Garcia \& Lisboa, 2012).

Ademais, a Atenção Primária à Saúde (APS) é um espaço onde os enfermeiros tem grande destaque na atuação à saúde da mulher. Segundo Souza et al., (2013), o cuidado de enfermagem é essencial na promoção à saúde, prevenção de doenças, na criação de vínculo, atendimento voltado às necessidades do indivíduo, a confiança e acolhimento prestado, resolutividade e sigilo profissional, tudo voltado a melhor atender e botar em prática o cuidado humanizado em saúde.

Souza et al., (2013), dizem que se faz necessário constantemente repensarmos a prática do atendimento com atualização em sexualidade. E está também no climatério, por ser tão peculiar, lembrando-se de focar nos aspectos e valores do que é intrínseco à esta mulher.

Garcia e Lisboa (2012) e Sehnem, et al., (2013) trouxeram à esclarecimento, estudos direcionados à sexualidade humana voltado ao cuidado da enfermagem e a de atendimento holístico e integral, que aborde o indivíduo como um todo, assim, atendendo os requisitos básicos da habilidade do cuidar. Deixando evidente que as pesquisas contribuem com a integralidade na assistência, quebrando tabus e educando a luz da ciência (Sehnem et al., 2013; Zampieri et al., 2009)

Fomentados pelos estudos que apontam os impasses aos profissionais de enfermagem, e que certamente prejudica a qualidade de assistência prestada, é importante refletirmos como esses profissionais entendem a relação do período do climatério e a sexualidade, fases de vivência da mulher.

O conhecimento sobre a sexualidade, climatério e o papel do enfermeiro diante deste cenário, reafirma que a base e essência do cuidar da enfermagem é constantemente ameaçada, e com isso, o surgimento de questionamentos sobre a habilidade e competência profissional para que haja solução no desenvolvimento das ações do saber científico (Sodson Da \& Silva, 2009). Determinada forma, tratar de assuntos delicados e de tal relevância exigem, do profissional o conhecimento das singularidades, que neste caso, relaciona-se com as áreas de conhecimento específico, onde os estudos apontam dificuldades na formação de forma prática e assistencial do cuidar.

Com base nisso, surgem os seguintes questionamentos: $\mathrm{O}$ enfermeiro entende as transformações sexuais e fisiológicas presentes no período do climatério? Compreende quais as alterações sexuais nesse momento da vida em que a mulher vivencia, e como ocorre a sexualidade? Como é compreendido o que a mulher expressa sobre as questões relacionadas à sexualidade? 
Em frente a tantos questionamentos, surge a necessidade deste estudo tendo como principal objetivo analisar o conhecimento do enfermeiro da Atenção primária sobre sexualidade da mulher no climatério e suas interfaces.

\section{Metodologia}

O presente estudo trata-se de uma revisão integrativa, que consiste nas abordagens metodológicas mais amplas entre as revisões de literatura. Segundo Souza, et al., (2017). a revisão permite a integração de estudos experimentais e não experimentais para que seja possível a compreensão completa do fenômeno estudado. A metodologia tem a finalidade de gerar síntese de como os resultados foram adquiridos nas pesquisas sobre um determinado tema, de forma sistemática e ordenada, concedendo informações amplas (Andrade, et al., 2017).

Fica evidente que a diversidade na composição da amostragem de uma revisão integrativa fortalece o conjunto de conceitos, teoria e problemas voltados aos cuidados de saúde, tornando-se um grande conjunto de alta relevância para a Enfermagem (Andrade, et al. 2017). Pensando assim foi formulada a seguinte pergunta norteadora: "Qual o papel do enfermeiro da APS na Atenção primária sobre sexualidade da mulher no climatério e suas interfaces?".

Com isso, as buscas foram realizadas nas bases de dados online Biblioteca Virtual em Saúde (BVS) e Scientific Electronic Library Online (SciELO), PUBMED e Google Acadêmico, através dos descritores "Sexualidade", "Climatério", "Atenção Primária à saúde", "Enfermagem", "Estratégia Saúde da Família”, por meio dos booleanos AND e OR. Foram utilizados como critérios de inclusão artigos que estivessem disponíveis no idioma Português, Inglês e Espanhol, datados nos últimos vinte anos e que abordassem o conhecimento do enfermeiro da Atenção primária sobre sexualidade da mulher no climatério e suas interfaces.

Esse recorte temporal foi preciso para melhor compreensão da temática, devido à escassez de material voltado ao conhecimento da enfermagem atuantes na atenção primária à saúde sobre a sexualidade no climatério. Foram excluídos os estudos que não se relacionavam com o tema proposto e não tinham o texto completo disponível na íntegra.

Diante disso, foram encontradas 873 publicações nas quais analisou-se os títulos e resumos, como também, os critérios de inclusão e exclusão proposto. Em seguida, 171 publicações foram pré-selecionadas para ser realizada a leitura na íntegra com o intuito de analisar criticamente os resultados obtidos. Após o processo de leitura e análise, 39 publicações foram selecionadas para compor a discussão e apresentação desta revisão integrativa. 
Figura 1. Fluxograma dos estudos selecionados adaptados do PRISMA. Recife, Pernambuco, Brasil.

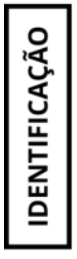

873 publicações identificadas a partir da busca das bases de dados.

SCIELO: 222; BVS: 125; PubMed: 14;

Google Acadêmico: 512.
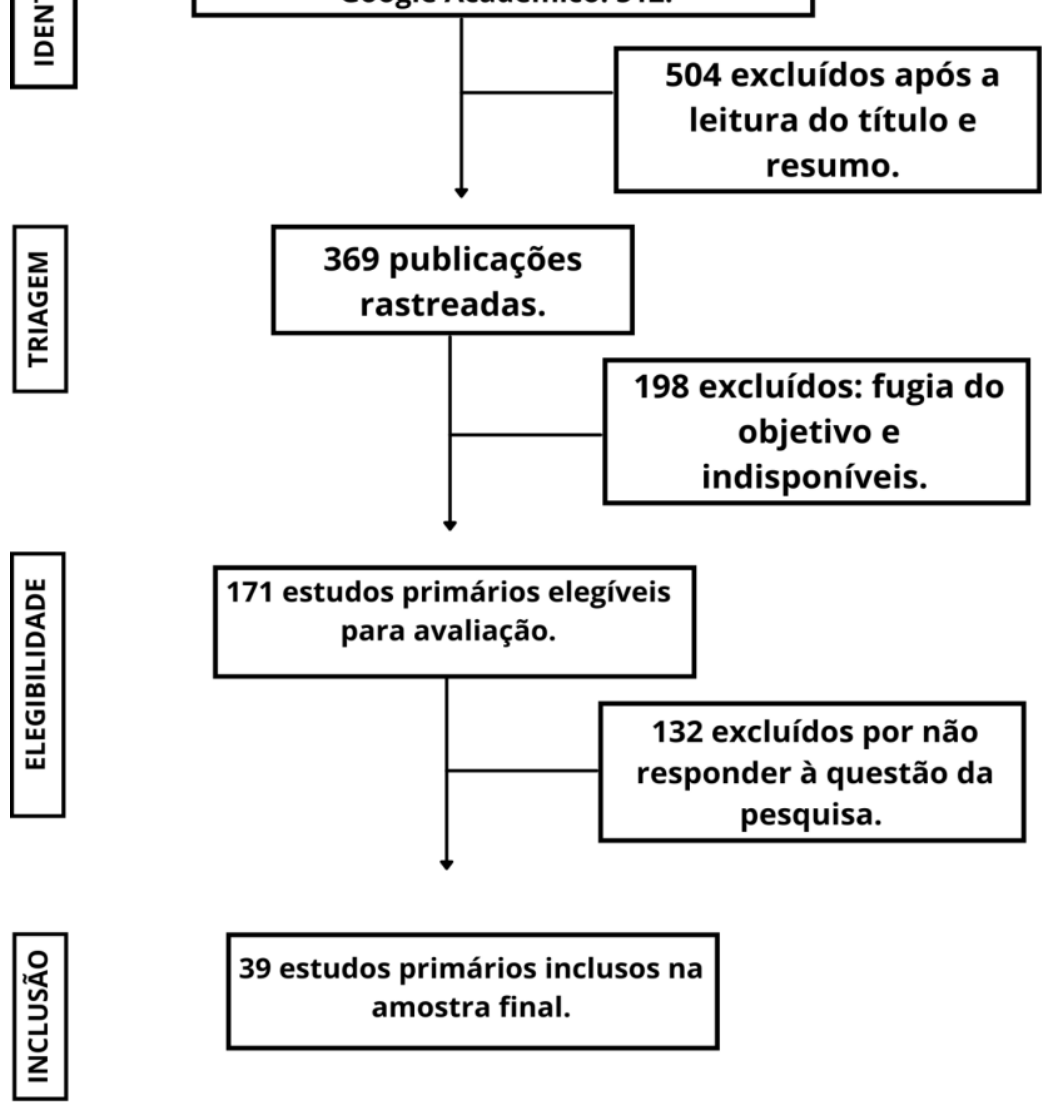

39 estudos primários inclusos na amostra final.

Fonte: Andrade, et al. (2021).

\section{Resultados e Discussão}

A amostra final desta revisão foi composta por 39 artigos científicos, datados nos últimos vinte anos de publicação e que abordassem a relação entre o conhecimento do enfermeiro da atenção primária sobre sexualidade da mulher no climatério e suas interfaces.

Nesse sentido, pode-se concluir o objetivo alvo adquirido dos artigos selecionados para leitura e de maior relevância na temática abordada, aplicando-os ao Quadro 1.

Quadro 1. Paramentação de produção científica com base nos achados científicos. Recife, Pernambuco, Brasil.

\begin{tabular}{|c|c|c|c|}
\hline Autores e periódico & Título & Objetivos & Resultados \\
\hline $\begin{array}{l}\text { Andréa Ramos da Silva, } \\
\text { Terezinha de Freitas Ferreira e } \\
\text { Ana Cristina d'Andretta } \\
\text { Tanaka. Rev. Bras. Cresc. e } \\
\text { Desenv. Hum. 2010; 20(3) 778- } \\
786 .\end{array}$ & $\begin{array}{l}\text { História ginecológica e } \\
\text { sintomatologia climatérica } \\
\text { de mulheres pertencentes a } \\
\text { uma unidade de saúde } \\
\text { pública do Estado do Acre. }\end{array}$ & $\begin{array}{l}\text { Descrever a história } \\
\text { ginecológica e a sintomatologia } \\
\text { de mulheres na pré, peri e pós } \\
\text { menopausa. }\end{array}$ & $\begin{array}{l}\text { As médias de idade da menarca, número de } \\
\text { gestações, número de filhos vivos e idade da } \\
\text { menopausa foram de } 13,3 \text { anos, } 3,5,2,8 \text { e } 47,5 \text { anos, } \\
\text { respectivamente. A diminuição do desejo sexual, a } \\
\text { tristeza, a dificuldade de concentração e o } \\
\text { esquecimento apresentaram associação com a fase } \\
\text { de perimenopausa }(p<0,05) \text {. A irritação e as ondas } \\
\text { de calor associaramse estatisticamente ( }<<0,05 \text { ) com } \\
\text { as fases de peri e pós-menopausa. }\end{array}$ \\
\hline
\end{tabular}




\begin{tabular}{|c|c|c|c|}
\hline $\begin{array}{l}\text { Maria de Fátima Mota Zampieri, } \\
\text { Celina Maria Araujo Tavares, } \\
\text { Maria de Lourdes Campos } \\
\text { Hames, Gladys Santos Falcon, } \\
\text { Alcione Leite de Silva e Lúcia } \\
\text { Takase Gonçalves. Esc. Anna } \\
\text { Nery, } 2009 .\end{array}$ & $\begin{array}{l}\text { O processo de viver e ser } \\
\text { saudável das mulheres no } \\
\text { climatério. }\end{array}$ & $\begin{array}{l}\text { Teve como objetivo } \\
\text { compreender como se dá o } \\
\text { processo de vida de mulheres no } \\
\text { climatério. }\end{array}$ & $\begin{array}{l}\text { O viver das mulheres no período do climatério } \\
\text { mostrou-se como um processo complexo, dinâmico, } \\
\text { paradoxal, em que o envelhecimento e a } \\
\text { possibilidade de adoecer se colocam como desafios } \\
\text { maiores e os avanços nas perspectivas pessoais, } \\
\text { culturais e sociais, como conquistas especiais. }\end{array}$ \\
\hline $\begin{array}{l}\text { Tito Sena. Estudos Feministas, } \\
\text { Florianópolis, } 2010 .\end{array}$ & $\begin{array}{l}\text { Os relatórios Masters \& } \\
\text { Johnson:gênero e as práticas } \\
\text { psicoterapêuticas sexuais a } \\
\text { partir da década de } 701 .\end{array}$ & $\begin{array}{l}\text { Este trabalho busca analisar os } \\
\text { relatórios Masters \& Johnson, } \\
\text { editados originalmente nos anos } \\
\text { de } 1966 \text { (A Resposta Sexual } \\
\text { Humana) e } 1970 \quad \text { (A } \\
\text { Inadequação Sexual Humana). }\end{array}$ & $\begin{array}{l}\text { Os relatórios analisados, produzidos nos Estados } \\
\text { Unidos e com repercussão mundial, foram } \\
\text { elaborados a partir de uma minuciosa investigação } \\
\text { científica das respostas fisiológicas e anatômicas da } \\
\text { sexualidade masculina e feminina. }\end{array}$ \\
\hline $\begin{array}{l}\text { Silvana Cruz da Silva, Caroline } \\
\text { Bolzan Ilha, Andrêssa Batista } \\
\text { Possati, Camila Neumaier } \\
\text { Alves, Camila Nunes Barreto, } \\
\text { Lúcia Beatriz Ressel, Láis } \\
\text { Antunes Wilhelm, Luiza } \\
\text { Cremoneles, Naiashy Vanuzzi, } \\
\text { Priscila Bisognin e Karine Eliel } \\
\text { Stumm. Convibra saúde. 2013. }\end{array}$ & $\begin{array}{lrr}\text { Análise da produção } & \text { do } \\
\text { conhecimento } & & \text { de } \\
\text { enfermagem } & \text { acerca } & \text { da } \\
\text { sexualidade } & & \text { dos } \\
\text { adolescentes. } & & \end{array}$ & $\begin{array}{l}\text { O objetivo geral da pesquisa é } \\
\text { analisar a produção do } \\
\text { conhecimento nacional sobre a } \\
\text { abordagem dos enfermeiros em } \\
\text { relação à sexualidade dos } \\
\text { adolescentes. }\end{array}$ & $\begin{array}{l}\text { Emergiram dois núcleos temáticos: a valorização da } \\
\text { família na construção da sexualidade dos } \\
\text { adolescentes; e a necessidade de estudos que } \\
\text { abordem as questões socioculturais relacionadas à } \\
\text { sexualidade dos adolescentes. }\end{array}$ \\
\hline $\begin{array}{l}\text { Olga Regina Zigelli Garcia e } \\
\text { Laura Cristina da Silva Lisboa. } \\
\text { Texto Contexto Enferm. } 2012 .\end{array}$ & $\begin{array}{l}\text { Consulta de enfermagem em } \\
\text { sexualidade: um instrumento } \\
\text { para assistência de } \\
\text { enfermagem à saúde da } \\
\text { mulher, em nível de atenção } \\
\text { primária. }\end{array}$ & $\begin{array}{lr}\text { Busca compreender } & \text { a } \\
\text { sexualidade como } & \text { um } \\
\text { componente da saúde. } & \end{array}$ & $\begin{array}{l}\text { Ao longo do texto buscam demonstrar a importância } \\
\text { da apropriação do conhecimento desta temática para } \\
\text { a atuação profissional do enfermeiro em nível de } \\
\text { atenção primária à saúde. }\end{array}$ \\
\hline $\begin{array}{l}\text { Graciela Dutra Sehnem, Lúcia } \\
\text { Beatriz Ressel, Carolina } \\
\text { Frescura Junges, Fernanda } \\
\text { Machado da Silva e Camila } \\
\text { Nunes Barreto. Esc. Anna Nery } \\
\text { Rev. Enferm. 2013. }\end{array}$ & $\begin{array}{l}\text { A sexualidade na formação } \\
\text { acadêmica do enfermeiro. }\end{array}$ & $\begin{array}{l}\text { Teve como objetivo analisar } \\
\text { como se dá a construção da } \\
\text { sexualidade na formação } \\
\text { acadêmica de estudantes de } \\
\text { Enfermagem. }\end{array}$ & $\begin{array}{l}\text { No que tange à formação acadêmica do enfermeiro } \\
\text { em relação à sexualidade, esse assunto tem sido } \\
\text { tratado a partir de um caráter de eventualidade e } \\
\text { informalidade e abordado sob um enfoque de } \\
\text { neutralidade, proibições e assexualização. }\end{array}$ \\
\hline $\begin{array}{l}\text { Tatiele Galli Zanetti, Isabel } \\
\text { Cristina Pacheco Van der Sand, } \\
\text { Nara Marilene Oliveira } \\
\text { Girardon-Perlini, Águida } \\
\text { Wicrowisky Kopf e Paola Braz } \\
\text { de Abreu. Cienc Cuid Saude, } \\
2010 .\end{array}$ & $\begin{array}{l}\text { Perfil socioprofissional e } \\
\text { formação de profissionais. }\end{array}$ & $\begin{array}{l}\text { O objetivo do estudo foi } \\
\text { caracterizar o perfil } \\
\text { socioprofissional e de formação } \\
\text { dos integrantes de equipes de } \\
\text { Saúde da Família. }\end{array}$ & $\begin{array}{l}\text { Constatou-se baixa rotatividade dos profissionais } \\
\text { nas equipes, o que pode qualificar a atenção } \\
\text { prestada, já que a permanência junto às comunidades } \\
\text { permite a formação de vínculo e favorece a interação } \\
\text { com elas. }\end{array}$ \\
\hline $\begin{array}{l}\text { Janielle Silva } \text { Fernandes, } \\
\text { Sybelle de Souza Castro } \\
\text { Miranzi, Helena Hemiko } \\
\text { Iwamoto, Darlene Mara dos } \\
\text { Santos Tavares e Claudia } \\
\text { Benedita dos Santos. Texto } \\
\text { Contexto Enferm, Florianópolis, } \\
\text { 2010. }\end{array}$ & $\begin{array}{l}\text { Qualidade de vida dos } \\
\text { enfermeiros das equipes de } \\
\text { saúde da família: a relação } \\
\text { das variáveis } \\
\text { sociodemográficas. }\end{array}$ & $\begin{array}{l}\text { Conhecer a qualidade de vida } \\
\text { dos enfermeiros que compõem } \\
\text { as equipes de saúde da família da } \\
\text { Macrorregião de Saúde do } \\
\text { Triângulo Sul, assim como } \\
\text { fatores sociodemográficos } \\
\text { relacionados }\end{array}$ & $\begin{array}{l}\text { Os resultados mostram o impacto negativo do estado } \\
\text { de saúde debilitado nos domínios da qualidade de } \\
\text { vida dos enfermeiros. }\end{array}$ \\
\hline $\begin{array}{l}\text { Maria de Fátima Mota } \\
\text { Zampieri; Celina Maria Araujo } \\
\text { Tavares; Maria de Lourdes } \\
\text { Campos Hames; Gladys Santos } \\
\text { Falcon; Alcione Leite de Silva; }\end{array}$ & $\begin{array}{l}\text { O processo de viver saudável } \\
\text { das mulheres no climatério. }\end{array}$ & $\begin{array}{l}\text { Compreender como se dá o } \\
\text { processo de viver de mulheres } \\
\text { no climatério. }\end{array}$ & $\begin{array}{l}\text { Emergiram as unidades de significado, que deram } \\
\text { origem às seguintes categorias: afirmando-se como } \\
\text { mulher; experienciando o climatério e o } \\
\text { envelhecimento; interagindo no cotidiano e } \\
\text { mantendo as singularidades; abrindo caminhos para }\end{array}$ \\
\hline
\end{tabular}




\begin{tabular}{|c|c|c|c|}
\hline $\begin{array}{l}\text { Lúcia Takase Gonçalves.Esc. } \\
\text { Anna Nery. Rio de Janeiro, } \\
2010 .\end{array}$ & & & a vivência da cidadania. \\
\hline $\begin{array}{l}\text { Antônio de Almeida Costa } \\
\text { Melo, Elania Pereira da Cruz } \\
\text { Silva, Ani Cátia Giotto.Rev Inic } \\
\text { Cient Ext. } 2019 .\end{array}$ & $\begin{array}{l}\text { Assistência da enfermagem à } \\
\text { mulher no climatério na } \\
\text { atenção básica de saúde. }\end{array}$ & $\begin{array}{l}\text { Objetiva-se as ações de } \\
\text { enfermagem na atenção básica } \\
\text { para auxiliar a mulher que } \\
\text { vivencia o climatério, } \\
\text { demonstrando a importância da } \\
\text { promoção de saúde, através da } \\
\text { consulta de enfermagem em } \\
\text { conhecer as estratégias } \\
\text { utilizadas acerca da atenção às } \\
\text { mulheres no período do } \\
\text { climatério }\end{array}$ & $\begin{array}{l}\text { A assistência da enfermagem na atenção básica é } \\
\text { um sistema complexo e relevante no âmbito do } \\
\text { gerenciamento dos sistemas e serviços de saúde, por } \\
\text { contemplar insumos básicos para cuidados aos } \\
\text { pacientes e pelos altos custos envolvidos. São } \\
\text { limitados os incentivos aos profissionais para } \\
\text { aprender sob atendimento em climatério e } \\
\text { capacitação aos funcionários das unidades. A } \\
\text { assistência realizada em face da mulher no } \\
\text { climatério na atenção básica de saúde contém } \\
\text { particularidades das quais se faz necessária o } \\
\text { conhecimento especializado, a legitimidade e o } \\
\text { reconhecimento das pacientes para com os } \\
\text { enfermeiros. }\end{array}$ \\
\hline $\begin{array}{l}\text { Olga Regina Zigelli } \\
\text { Garcia,Laura Cristina da Silva } \\
\text { Lisboa.Texto Contexto Enferm. } \\
\text { 2012. }\end{array}$ & $\begin{array}{l}\text { Consulta de enfermagem em } \\
\text { sexualidade: um instrumento } \\
\text { para assistência de } \\
\text { enfermagem à saúde da } \\
\text { mulher, em nível de atenção } \\
\text { primária. }\end{array}$ & $\begin{array}{l}\text { Buscam demonstrar a } \\
\text { importância da apropriação do } \\
\text { conhecimento desta temática } \\
\text { para a atuação profissional do } \\
\text { enfermeiro em nível de atenção } \\
\text { primária à saúde }\end{array}$ & $\begin{array}{l}\text { Aprofundar-se no estudo da sexualidade humana, em } \\
\text { especial a feminina, é uma das demandas na } \\
\text { formação e atuação de profissionais enfermeiros }\end{array}$ \\
\hline $\begin{array}{l}\text { Graciela Dutra Sehnem, Lúcia } \\
\text { Beatriz Ressel, Carolina } \\
\text { Frescura Junges, Fernanda } \\
\text { Machado da Silva,Camila } \\
\text { Nunes Barreto.Esc Anna Nery } \\
\text { Rev Enferm [Internet]. 2013. }\end{array}$ & $\begin{array}{l}\text { A sexualidade na formação } \\
\text { acadêmica do enfermeiro. }\end{array}$ & $\begin{array}{ll}\text { Analisar como se dá a } \\
\text { construção da sexualidade na } \\
\text { formação acadêmica } & \text { de } \\
\text { estudantes de Enfermagem. } & \end{array}$ & $\begin{array}{l}\text { No que tange à formação acadêmica do enfermeiro } \\
\text { em relação à sexualidade, esse assunto tem sido } \\
\text { tratado a partir de um caráter de eventualidade e } \\
\text { informalidade e abordado sob um enfoque de } \\
\text { neutralidade, proibições e assexualização. }\end{array}$ \\
\hline $\begin{array}{l}\text { Marcia Goulart de Souza;Edir } \\
\text { Nei Teixeira Mandu;Alessandra } \\
\text { Nogueira Elias.Cienc Cuid } \\
\text { Saude, 2010. }\end{array}$ & $\begin{array}{l}\text { Percepções de enfermeiros } \\
\text { sobre a Estratégia Saúde da } \\
\text { Família }\end{array}$ & $\begin{array}{l}\text { Analisar percepções de } \\
\text { enfermeiros sobre o seu trabalho } \\
\text { na Estratégia Saúde da Família. }\end{array}$ & $\begin{array}{l}\text { O trabalho do enfermeiro é concebido a partir de } \\
\text { ações estratégicas e da prevenção, da atuação } \\
\text { assistencial em contraponto à atuação gerencial, e da } \\
\text { interação acolhedora com usuários. Localiza-se } \\
\text { aderência a eixos propostos para o trabalho na } \\
\text { Estratégia Saúde da Família e certo } \\
\text { desconhecimento ou absorção acrítica destes, } \\
\text { sobretudo no que diz respeito à promoção da saúde e } \\
\text { à renovação da prática gerencial local. }\end{array}$ \\
\hline $\begin{array}{l}\text { Tatiele Galli Zanetti; Isabel } \\
\text { Cristina Pacheco Van der Sand; } \\
\text { Nara Marilene Oliveira } \\
\text { Girardon-Perlini; Águida } \\
\text { Wicrowisky Kopf; Paola Braz } \\
\text { de Abreu.Cienc. Cuid. Saúde, } \\
\text { 2010a. }\end{array}$ & $\begin{array}{l}\text { Perfil socioprofissional e } \\
\text { formação de profissionais. }\end{array}$ & $\begin{array}{l}\text { Caracterizar o perfil } \\
\text { socioprofissional e de formação } \\
\text { dos integrantes de equipes de } \\
\text { Saúde da Família (ESF) do } \\
\text { Interior do Rio Grande do Sul, } \\
\text { no Noroeste do Estado, } \\
\text { especificamente da } 14^{\text {a }} \\
\text { Coordenadoria Regional de } \\
\text { Saúde. }\end{array}$ & $\begin{array}{l}\text { Constatou-se baixa rotatividade dos profissionais } \\
\text { nas equipes, o que pode qualificar a atenção } \\
\text { prestada, já que a permanência junto às comunidades } \\
\text { permite a formação de vínculo e favorece a interação } \\
\text { com elas. Quanto à formação dos trabalhadores, há } \\
\text { iniciativas para a realização de cursos, } \\
\text { especializações e/ou residências, o que tende a } \\
\text { refletir-se na atenção básica, no cuidado às pessoas e } \\
\text { suas famílias e no seguimento da estratégia de saúde } \\
\text { e do processo de trabalho. }\end{array}$ \\
\hline $\begin{array}{l}\text { Amanda Carla dos Santos } \\
\text { Beltramini } \quad \text {;Christiane } \\
\text { Aparecida Paschoal Diez; Iara } \\
\text { Orlando Camargo; Vivian Aline } \\
\text { Preto. Rev. Min. Enferm., } 2010 .\end{array}$ & $\begin{array}{l}\text { Atuação do Enfermeiro } \\
\text { diante da importância da } \\
\text { assistência à saúde da mulher } \\
\text { no climatério. }\end{array}$ & $\begin{array}{l}\text { Verificar o conhecimento dos } \\
\text { enfermeiros diante da definição } \\
\text { de climatério, descrever o } \\
\text { planejamento específico de } \\
\text { enfermeiros para a assistência à } \\
\text { mulher no climatério, bem como } \\
\text { a importância que os } \\
\text { enfermeiros oferecem à atenção } \\
\text { para estas mulheres. }\end{array}$ & $\begin{array}{l}\text { O estudo permitiu ressaltar a extrema importância } \\
\text { de o enfermeiro se munir de informações a respeito } \\
\text { do tema para ter condições de oferecer uma } \\
\text { assistência adequada, refletir sobre o assunto e se } \\
\text { conscientizar da necessidade da prática de tais ações. }\end{array}$ \\
\hline $\begin{array}{lrr}\text { Neide } & \text { Aparecida } & \text { Titonelli } \\
\text { Alvim, Márcia de } & \text { Assunção } \\
\text { Ferreira.Texto } & \text { Contexto } \\
\text { Enfermagem, } & \text { Florianópolis, } \\
\text { 2007. } & \end{array}$ & $\begin{array}{l}\text { Perspectiva } \\
\text { Problematizadora da } \\
\text { Educação Popular em Saúde } \\
\text { e Enfermagem. }\end{array}$ & $\begin{array}{l}\text { Refletir acerca da educação } \\
\text { popular em saúde no contexto do } \\
\text { educar-cuidar da enfermeira, em } \\
\text { uma perspectiva crítica e } \\
\text { problematizadora. }\end{array}$ & $\begin{array}{l}\text { As idéias freireanas, aplicadas à prática educativa da } \\
\text { enfermeira, possibilitam pela crítica e reflexão, a } \\
\text { transformação de saberes dentro de um grupo que } \\
\text { não tem o conhecimento advindo da ciência, ao } \\
\text { mesmo tempo em que também nos apropriamos do } \\
\text { conhecimento que vem do universo do senso } \\
\text { comum. Nessa perspectiva, a pessoa tem a } \\
\text { oportunidade de pontuar e refletir sobre os próprios } \\
\text { veículos da educação em saúde. }\end{array}$ \\
\hline
\end{tabular}




\begin{tabular}{|c|c|c|c|}
\hline $\begin{array}{l}\text { Cecília Nogueira Valença,José } \\
\text { Medeiros do Nascimento } \\
\text { Filho,Raimundar Medeiros } \\
\text { Germano.Saúde social. } 2010 .\end{array}$ & $\begin{array}{lrr}\text { Mulher no } & \text { climatério: } \\
\text { reflexões } & \text { sobre } & \text { desejo } \\
\text { sexual, } & \text { beleza } & \text { e } \\
\text { feminilidade. } & & \end{array}$ & $\begin{array}{l}\text { Refletir sobre desejo sexual, } \\
\text { beleza e feminilidade da mulher } \\
\text { nessa fase. }\end{array}$ & $\begin{array}{l}\text { A exigência exacerbada pela beleza eterna } \\
\text { jovialidade é agravada no climatério, no qual o corpo } \\
\text { feminino não tem o mesmo vigor físico pelas } \\
\text { alterações decorrentes do envelhecimento. A mulher } \\
\text { climatérica vive o mito da perda do desejo sexual, } \\
\text { todavia, continua a sentir prazer, não devendo deixar } \\
\text { de manifestar amor e sexualidade. A visão social } \\
\text { estereotipada sobre o papel da mulher (esposa e mãe) } \\
\text { pode interferir negativamente na visão das mulheres } \\
\text { sobre si mesmas e no seu relacionamento com as } \\
\text { pessoas e o mundo. }\end{array}$ \\
\hline $\begin{array}{l}\text { Dino Roberto Soares De } \\
\text { Lorenzi, Bruno SacilotoS. Rev. } \\
\text { Assoc. Med. Bras., São Paulo, v. } \\
\text { 52, n. 4, Aug. 2006. }\end{array}$ & $\begin{array}{ll}\text { Frequência da } & \text { atividade } \\
\text { sexual em } & \text { mulheres } \\
\text { menopausadas. } & \end{array}$ & $\begin{array}{lll}\text { Identificar } & \text { os fatores } \\
\text { relacionados à } & \text { freqüência da } \\
\text { atividade sexual entre mulheres } \\
\text { pós-menopáusicas }\end{array}$ & $\begin{array}{l}\text { Das mulheres pesquisadas, } 176(85 \%) \text { eram } \\
\text { sexualmente ativas. Cerca de } 60,6 \% \text { relataram } \\
\text { diminuição da atividade sexual após a menopausa, } \\
\text { que atribuíram principalmente à impotência sexual } \\
\text { do parceiro }(41,7 \%) \text {. Aproximadamente } 25,7 \% \\
\text { negaram satisfação com o intercurso sexual. Na } \\
\text { análise por regressão linear múltipla, associaram-se } \\
\text { à atividade sexual a idade ( }<0,01) \text {, o grau de } \\
\text { satisfação sexual ( } p=0,01) \text { e a sintomatologia } \\
\text { climatérica }(p=0,02) \text {. Quanto maior a idade, mais } \\
\text { intensa a sintomatologia climatérica, menor a } \\
\text { satisfação sexual e menos freqüente a atividade } \\
\text { sexual. Os sintomas climatéricos que se } \\
\text { correlacionaram com a atividade sexual foram os } \\
\text { fogachos ( } p=0,05) \text { a irritabilidade ( } p=0,04) \text { a a } \\
\text { melancolia/tristeza }(p=0,04) \text {, as artralgias/mialgias } \\
(p<0,01) \text { e a fraqueza/cansaço }(p<0,01) \text {. }\end{array}$ \\
\hline $\begin{array}{l}\text { Lúcia Alves da Silva Lara; Ana } \\
\text { Carolina Japur de Sá Rosa e } \\
\text { Silva; Adriana Peterson } \\
\text { Mariano Salata Romão; Flavia } \\
\text { Raquel Rosa Junqueira.Rev. } \\
\text { Bras. Ginecol. Obstet. } 2008 .\end{array}$ & $\begin{array}{l}\text { Abordagem das disfunções } \\
\text { sexuais femininas. }\end{array}$ & $\begin{array}{l}\text { Exemplificação do modelo } \\
\text { PILSET (PLISSIT), uma técnica } \\
\text { de abordagem da função sexual } \\
\text { humana, é composto por quatro } \\
\text { elementos: permissão, } \\
\text { informação limitada, sugestão } \\
\text { específica e terapia sexual, que } \\
\text { favorecem o diálogo entre o } \\
\text { médico e a paciente, permitindo } \\
\text { o acesso às queixas sexuais. }\end{array}$ & $\begin{array}{l}\text { Traz como usar o modelo PILSET. Adicionalmente, } \\
\text { vários aspectos da função sexual feminina, como } \\
\text { prevalência, diagnóstico e outras modalidades de } \\
\text { tratamento são discutidos. }\end{array}$ \\
\hline
\end{tabular}

Fonte: Andrade, et al. (2021).

A fase da climatério é discutida como o processo de transição do período reprodutivo e o não reprodutivo da mulher (Lavôr, et al., 2010). A característica das mudanças hormonais, morfológicas e pelo esgotamento de folículos ovarianos, pode afetar de forma negativa a vida da mulher (Carla et al., 2010). Ainda com Carla et al., (2010), o climatério afeta diretamente a qualidade de vida sexual e psicológica, e a relação do enfermeiro está ligada devido ao contato regular com as mulheres ao longo da vida.

\section{Conhecimentos dos enfermeiros da APS sobre sexualidade}

A sexualidade ainda trabalhada com estigmas e tabus na cultura popular, fecha espaços de importante diálogo e debate, apesar de todos os avanços acadêmicos, sociais e midiáticos (N. C. M. da Silva et al., 2021). Garcia e Lisboa (2012) trazem em seu relatos a necessidade do profissional de enfermagem trabalha a temática da sexualidade de forma clara, onde se tem o destaque: empatia e habilidade - necessidade do paciente e mostrar sua compreensão; congruência - ser lógico na interação; aceitação - respeito pela sexualidade e individualidade do outro; e concreticidade - buscar soluções. Essas habilidades ressaltam a cada indivíduo o direito de definição longe de julgamento, a sua própria identidade sexual e sua realização.

De acordo com De Lorenzi e Sacilito (2006), juntamente com Lara, et al., (2008), é necessário que o profissional de enfermagem seja holístico as múltiplas necessidades do ser humano, na qual veja o indivíduo integralmente, seja atento na história de vida e o contexto onde está inserido buscando respeitar as crenças e os valores de cada cultura. Zampieri, et al., (2009) traz que a educação em saúde com parceria entre usuário e o profissional é o caminho de autoconhecimento. 


\section{Estratégia na assistência de enfermagem na APS}

Ferreira et al., (2021) debatem que o vínculo com a comunidade são ferramentas chaves no apoio e aprimoramento do profissional de Enfermagem. Indubitavelmente, a enfermagem como profissão que estima o compromisso com a população, objetiva sempre buscar a melhoria de qualidade de vida e assistência com base na ciência, que busca novas metodologias para melhor atender as necessidades do indivíduo, mediante atividades educativas em saúde e intervenções necessárias. As mulheres são maioria entre os usuários do SUS, onde, mesmo com todas as demandas e dificuldades diárias, essas mulheres buscam cuidados básicos à saúde. A educação em saúde é um ponto chave na implementação de cuidados (Alvim \& Ferreira, 2007). Cabendo ao enfermeiro da Estratégia de Saúde da Família (ESF) buscar um meio viável na criação de vínculo e facilitação ao atendimento, melhoria da qualidade de vida e cuidados integrais ao indivíduo (Brasil, 2009).

Em análise dos relatos de Sodson Da e Silva., (2009), é entendido como contribuição da Enfermagem em relação à autonomia da mulher climatérica. A APS tem como uma das demandas, fazer a implementação de uma estratégia voltada ao conforto na assistência à mulher, buscando criar vínculos e tornar o atendimento mais personalizado. No processo de atenção à saúde da mulher no climatério, é essencial atentar se a qualidade de vida das mulheres e como elas são afetadas diretamente pelas questões socioeconômicas (Fernandes, et al., 2010). Ainda com Fernandes, et al., (2010) as mulheres chefes de família e que têm mais de dois empregos tendem a não darem atenção às necessidades básicas, como sua saúde ou sexualidade.

\section{Conclusão}

A importância da assistência de enfermagem à mulher no climatério engloba desde o cuidado do corpo com propriedade e intimidade, abarcando os aspectos mais elementares do cuidado como a higiene corporal até os mais complexos, como identificar inadequações e disfunções sexuais e, assim, prescrever os cuidados de enfermagem. Sabe-se que a sexualidade ainda é permeada por diversos estigmas e tabus, sendo importante que o profissional de enfermagem aborda essa questão de forma clara e empática para que essa mulher possa ter o direito de usufruir da sua sexualidade livre de julgamentos, com a sua própria identidade sexual e se sinta realizada.

No âmbito da ESF, o profissional de enfermagem deve tratar a sexualidade como um dos pilares da qualidade de vida e criar um vínculo com a mulher, acolhendo-a e empoderando-a, enfatizando que é mais uma fase da vida e com os cuidados apropriados consegue vivenciá-la com menos dificuldades e mais fortalecida. Além disso, é importante compreender a realidade socioeconômica na qual a mulher está inserida, tendo em vista que a qualidade de vida é diretamente afetada por esse fator, incluindo acesso à saúde e a orientação sobre sexualidade. Nesse contexto, o enfermeiro irá atuar com estratégias que visem a criação de um vínculo entre essa mulher e a unidade de saúde, tornando possível uma assistência integral e focalizada nas suas necessidades e utilizando da educação em saúde como uma ferramenta que proporciona o autoconhecimento.

Desse modo, os achados e reflexões apresentados neste estudo podem contribuir para a melhoria da assistência prestada às mulheres no climatério pelos profissionais de enfermagem na Estratégia Saúde da Família. Como também, aponta-se a importância de pesquisas que explorem mais a atuação da assistência de Enfermagem à mulher no climatério, tendo em vista a importância desse profissional durante essa fase da vida.

\section{Referências}

Alvim, N. A. T., \& Ferreira, M. de A. (2007). Perspectiva problematizadora da educação popular em saúde e a enfermagem. Texto \& Contexto - Enfermagem, $16(2), 315-319$.

Andrade, S. R. de, Ruoff, A. B., Piccoli, T., Schmitt, M. D., Ferreira, A., \& Xavier, A. C. A. (2017). O Estudo de caso como método de pesquisa em enfermagem: uma revisão integrativa. Texto \& Contexto - Enfermagem, 26(4).

Brasil. Ministério da Saúde. (2009) Avaliação para Melhoria da Qualidade da Estratégia Saúde da Família. Caderno de Auto-Avaliação 4. https://bvsms.saude.gov.br/bvs/publicacoes/caderno_autoavaliacao_n4_saude_familia_parte1.pdf 
Brasil Ministério da saúde. (2008). Manual de Atenção à Mulher no Climatério / Menopausa.

Brasil. Ministério da Saúde. (2013). Saúde sexual e saúde reprodutiva. Cadernos de Atenção Básica, $n .26$.

Brasil. Ministério da Saúde. Política Nacional de Atenção Integral à Saúde da Mulher: Princípios e Diretrizes / Ministério da Saúde, Secretaria de Atenção à Saúde, Departamento de Ações Programáticas Estratégicas. Editora do Ministério da Saúde, 2011.

Brasil. Ministério da Saúde. (2006). Cadernos de atenção básica envelhecimento e saúde da pessoa idosa. http://189.28.128.100/dab/docs/publicacoes/cadernos_ab/abcad19.pdf

Carla, A. et al. (2010). Atuação do enfermeiro diante da importância da assistência à saúde da mulher no climatério the nurse actions regarding the importance of women healthcare in climacteric actitud del enfermero ante la importancia de la atención a la salud de la mujer en el climaterio. In remE-Rev. Min. Enferm 14(2).

Cruz da Silva, S. et al. (2013). Análise da produção do conhecimento de enfermagem acerca da sexualidade dos adolescentes. Convibra Saúde.

de Lorenzi, D. R. S., \& Saciloto, B. (2006). Freqüência da atividade sexual em mulheres menopausadas. Revista Da Associação Médica Brasileira, 52(4), 256260 .

Fernandes, J. S., Miranzi, S. de S. C., Iwamoto, H. H., Tavares, D. M. dos S., \& Santos, C. B. dos. (2010). Qualidade de vida dos enfermeiros das equipes de saúde da família: a relação das variáveis sociodemográficas. Texto \& Contexto - Enfermagem, 19(3), 434-442.

Ferreira, D. S., Ramos, F. R. S., \& Teixeira, E. (2021). Nurses' educational practices in Family Health Strategy. Revista Brasileira de Enfermagem, 74(2).

Garcia, O. R. Z., \& Lisboa, L. C. da S. (2012). Consulta de enfermagem em sexualidade: um instrumento para assistência de enfermagem à saúde da mulher, em nível de atenção primária. Texto \& Contexto - Enfermagem, 21(3), 708-716. https://doi.org/10.1590/S0104-07072012000300028

Gonçalves, E. (2021). Sexualidade no envelhecimento e a atuação do enfermeiro na atenção primária à saúde: revisão narrativa. (Trabalho de conclusão de curso). PUC-GOIÁS. Goiânia, GO, Brasil.

Lara, L. A. da S., Silva, A. C. J. de S. R. e, Romão, A. P. M. S., \& Junqueira, F. R. R. (2008). Abordagem das disfunções sexuais femininas. Revista Brasileira de Ginecologia e Obstetrícia, 30(6), 312-321. https://doi.org/10.1590/S0100-72032008000600008

LAVÔR, R. M. de. (2010). Estratégia de saúde da família na assistência à mulher na fase do climatério. Trabalho de Conclusão de Curso (Bacharelado em Enfermagem) - Centro de Formação de Professores, Universidade Federal de Campina Grande, Cajazeiras, Paraíba, Brasil.

Maciel, J. B. L., Sipaúba, A. J. C., Andrade, T. L. da C., Barroso, H. L. M. R., Amorim, J. F. de, Silva, K. de S. M. da, \& Souza, A. da S. (2021). Vivência e concepção da mulher acerca do climatério: Uma revisão bibliográfica. Research, Society and Development, $10(6)$, e9710615557.

Melo, A. de A. C., Silva, E. P. da C., \& Giotto, A. C. (2019). Assistência da enfermagem à mulher no climatério na atenção básica de saúde. Revista De Iniciação Científica Extensão, 2(4), 213-218.

Nogueira, A. J. da S., \& Pachú, C. O. (2021). Sexualidade da mulher e autocuidado no âmbito da Atenção Primária à Saúde: uma revisão integrativa. Research, Society and Development, 10(15), e95101522157.

Pontes, A. F., Sarmento, B. C. dos S., Moura, A. C. Q. da L., Tavares, C. M. de A., Silva, S. R. C. da, Deodoro, M. F. P., Leuthier, K. de H., Silva, B. C. da, Rodrigues, N. A., \& Andrade, Â. R. L. de. (2021). Sexualidade feminina em tempos de pandemia da COVID-19. Research, Society and Development, 10(12), e494101220146.

Sehnem, G. D., Ressel, L. B., Junges, C. F., Silva, F. M. da, \& Barreto, C. N. (2013). A sexualidade na formação acadêmica do enfermeiro. Escola Anna Nery, 17(1), 90-96. https://doi.org/10.1590/S1414-81452013000100013

Sena, T. (2010). Os relatórios Masters \&amp; Johnson: gênero e as práticas psicoterapêuticas sexuais a partir da década de 70 . Revista Estudos Feministas, $18(1), 221-240$

Silva, A. R. da, Ferreira, T. de F., \& Tanaka, A. C. d'Andretta. (2010). História ginecológica e sintomatologia climatérica de mulheres pertencentes a uma unidade de saúde pública do Estado do Acre. Journal of Human Growth and Development, 20(3), 778-786.

Silva, N. C. M. et al. (2021). Sexuality and assessment of physical and psychological symptoms of older adults in outpatient care. Revista Brasileira de Enfermagem, 74(suppl 2).

Sodson Da, A., \& Silva, R. (2009). Assistência realizada por enfermeiros do psf a mulher no climatério. Caderno de Cultura e Ciência. Ano IV - Vol. 1- No 1.

Sousa, L. M. M. et al. (2017). Metodologia de Revisão Integrativa da Literatura em Enfermagem. Rev. Investigação em Enf. p.17-26.

Souza, M. G. de, Mandu, E. N. T., \& Elias, A. N. (2013). Percepções de enfermeiros sobre seu trabalho na Estratégia Saúde da Família. Texto \& Contexto Enfermagem, 22(3), 772-779.

Valença, C. N., Nascimento Filho, J. M. do, \& Germano, R. M. (2010). Mulher no climatério: reflexões sobre desejo sexual, beleza e feminilidade. Saúde e Sociedade, 19(2), 273-285.

Zampieri, M. de F. M., Tavares, C. M. A., Hames, M. de L. C., Falcon, G. S., Silva, A. L. de, \& Gonçalves, L. T. (2009). O processo de viver e ser saudável das mulheres no climatério. Escola Anna Nery, 13(2), 305-312.

Zanetti, T. G., van der Sand, I. C. P., Girardon-Perlini, N. M. O., Kopf, Á. W., \& Abreu, P. B. de. (2010). Perfil socioprofissional e formação de profissionais de equipes de saúde da família: um estudo de caso. Ciência, Cuidado e Saúde, 9(3). 